\title{
The approach to coronary bifurcation treatment and its outcomes in Poland: The single center experience
}

\author{
Adam Kern ${ }^{1,2}$, Robert J. Gil ${ }^{3,4}$, Krystian Bojko², Bartłomiej Rzeszowski², \\ Rakesh Jalali1 ${ }^{1,5}$, Jerzy Górny², Jacek Bil ${ }^{3}$
}

${ }^{1}$ Faculty of Medical Sciences, University of Warmia and Mazury, Olsztyn, Poland

${ }^{2}$ Department of Cardiology, Regional Specialist Hospital, Olsztyn, Poland

${ }^{3}$ Department of Invasive Cardiology, Central Clinical Hospital of the Ministry of Interior, Warsaw, Poland ${ }^{4}$ Institute of Experimental and Clinical Medicine, Polish Academy of Science, Warsaw, Poland

${ }^{5}$ Emergency Medicine Department, Regional Specialist Hospital, Olsztyn, Poland

\begin{abstract}
Background: Coronary bifurcation lesions pose therapeutic problems during percutaneous coronary interventions. The aim of this study was to analyze the strategy of coronary bifurcation treatment and associated angiographic as well as clinical outcomes in a large hospital in Northern Poland.

Methods: Between January 2012 and January 2014 patients with stable coronary artery disease or non-ST-elevation acute coronary syndrome (NSTE-ACS) were treated with regular drug-eluting stents (rDES) or dedicated bifurcation stents (BiOSS Expert ${ }^{\circledast}$ or BiOSS LIM ${ }^{\circledR}$ ). Clinical and angiographic controls were planned at 12 months. The primary endpoint was major adverse cardiovascular events (MACE) rate composed of cardiac death, myocardial infarction, and target lesion revascularization (TLR) at 12 months.

Results: In total, 152 patients were enrolled in whom 158 stents were deployed (99 BiOSS stents and $59 \mathrm{rDES})$. Left anterior descending artery (50\%) was the dominant target vessel followed by left circumflex (25\%). There was no stent implantation failure. In 10 (6.3\%) patients rDES was required within the side branch. At 12 months MACE rate was $11.2 \%$, whereas TLR rate was $7.9 \%$. In the logistic regression analysis final kissing balloon technique was the prognostic factor for better clinical outcome, whereas NSTE-ACS and true bifurcations were risk factors of a poor outcome.

Conclusions: Percutaneous coronary bifurcation treatment is a safe and effective procedure, and provisional T-stenting is the preferred technique. Both rDES as well as dedicated bifurcation stents enabled a simple and fast bifurcation treatment option with comparable MACE and TLR rates. (Cardiol J 2017; 24, 6: 589-596)
\end{abstract}

Key words: BiOSS, culotte technique, dedicated bifurcation stent, provisional T-stenting

\section{Introduction}

Coronary bifurcation lesions pose a therapeutic challenge and are linked with higher rates of periprocedural complications as well as higher rates of in-stent restenosis and stent thrombosis [1]. Presently, provisional T-stenting (PTS) is the best approach [2, 3]. However, the optimal strategy for coronary bifurcations treatment remains a subject of debate, mainly when the side branch (SB) is large, not easily accessible or narrowed by a long lesion [4-7].

The aim of this study was to analyze the strategy for coronary bifurcation lesion treatment

Address for correspondence: Adam Kern, MD, PhD, Faculty of Medical Sciences, University of Warmia and Mazury, ul. Żołnierska 18, 10-561 Olsztyn, Poland, e-mail: adamkern@mail.com 
and associated clinical as well as angiographic outcomes in a large hospital in Northern Poland.

\section{Methods}

\section{Study population and study plan}

It was a prospective registry conducted between January 2012 to January 2014 in a highvolume center ( $>1500$ percutaneous coronary intervention $[\mathrm{PCI}]$ per year) in Poland (Olsztyn). Patients with stable coronary artery disease (CAD) or non-ST-segment elevation acute coronary syndrome (NSTE-ACS) were considered eligible for enrollment. The inclusion criteria were: age $\geq 18$ years old, de novo coronary bifurcation lesion (including unprotected left main), main vessel (MV) diameter $\geq 2.5 \mathrm{~mm}$ and $\mathrm{SB}$ diameter $\geq 2.0 \mathrm{~mm}$ assessed by visual estimation. Main exclusion criteria were: ST-elevation myocardial infarction (STEMI), inability to take dual antiplatelet therapy for 12 months, left ventricular ejection fraction $\leq$ $30 \%$ as well as lack of written informed consent. Institutional Review Board approved the study protocol.

\section{Interventional procedure and concomitant medications}

Procedures were performed by six independent operators. Single stent implantation in the proximal MV-distal MV across SB was the default strategy in all patients (PTS). Bifurcation lesions were assessed according to Medina classification using an index of 1 for stenosis greater than $50 \%$ and 0 for no stenosis (visual estimation). There was no restriction regarding lesion length in patient selection. The main indication for using dedicated bifurcation stents was the ratio of proximal MV diameter to distal MV diameter $>1.2$. If required, additional regular drug-eluting stents (rDES) were implanted. A stent in SB was implanted only if proximal residual stenosis was greater than $70 \%$ after balloon dilatation and/or significant flow impairment after proximal MV-distal MV stenting and/or a flow limiting dissection were noted. The implantation protocol was as follows:

1. Wiring of both branches;

2. MV predilatation and/or SB predilatation according to the operator's decision;

3. Stent implantation (inflation for at least $20 \mathrm{~s}$ );

4. Proximal optimization technique (POT) at operator's discretion;

5. SB postdilatation/stent implantation if necessary;

6. Final kissing balloons (FKB) inflation at operator's discretion.
In patients with NSTE-ACS, a loading dose of clopidogrel $(600 \mathrm{mg})$, ticagrelor $(180 \mathrm{mg}$ ) or prasugrel (60 mg) was given, and, if needed, also a loading dose of acetylsalicylic acid (ASA) was applied (300 mg). In planned procedures, $72 \mathrm{~h}$ before PCI each patient received ASA (75 mg/24 h) and clopidogrel $(75 \mathrm{mg} / 24 \mathrm{~h})$. All procedures were performed in a standard way via radial or femoral access using 6 Fr or 7 Fr guiding catheters. After insertion of the arterial sheath each patient received unfractionated heparin (70-100 IU/kg). Additional bolus was given to maintain an activated clotting time $>250 \mathrm{~s}$. Dual antiplatelet therapy (ASA $75 \mathrm{mg}$ q.d. and clopidogrel $75 \mathrm{mg}$ q.d., prasugrel $10 \mathrm{mg}$ q.d. or ticagrelor $90 \mathrm{mg}$ b.i.d.) was prescribed for 12 months.

All patients had troponin I (TnI), creatinine kinase (CK) and CK-MB levels examined before the procedure, $6 \mathrm{~h}$ and $24 \mathrm{~h}$ thereafter. Periprocedural myocardial infarction (MI) (type 4a) was assessed according to the third universal definition [8].

\section{Device description}

All drug-eluting stents available in the cathlab could have been used. In this study there were regular paclitaxel-eluting stents LucChopin2 with strut thickness of $120 \mu \mathrm{m}$, sirolimus-eluting stents Alex with strut thickness of $70 \mu \mathrm{m}$ or Cre 8 with strut thickness of $80 \mu \mathrm{m}$ and everolimus-eluting stents Xience with strut thickness of $81 \mu \mathrm{m}$ and two dedicated bifurcation stents: paclitaxel-eluting BiOSS Expert ${ }^{\circledR}$ with strut thickness of $120 \mu \mathrm{m}$ and sirolimus-eluting BiOSS LIM $^{\circledast}$ with strut thickness of $120 \mu \mathrm{m}[9,10]$.

\section{Follow-up}

Clinical follow-up was performed with office visits or telephone contact at 12 months after intervention. Adverse events were monitored throughout the study period. Follow-up coronary angiography was performed at 12 months unless clinically indicated earlier.

\section{Endpoints}

The primary endpoint was the cumulative rate of major adverse cardiovascular events (MACE) including cardiac death, MI and repeated revascularization of the target lesion (TLR). The secondary endpoints included cardiac death, all-cause death, MI, TLR and late lumen loss (LLL). All deaths were deemed cardiac unless proven otherwise.

\section{Angiographic analysis}

All angiograms were recorded after intracoronary administration of nitroglycerin $(200 \mu \mathrm{g})$. Two orthogonal views were chosen to visualize the 
target lesion. A quantitative coronary angiography (QCA) was performed using dedicated bifurcation software CAAS version 5.9 (2D analysis). Catheter calibration was performed in all cases. The proximal main vessel (the artery before SB take-off), the distal main vessel (artery beyond the ostium of SB), and the SB (the smaller vessel at the point of vessel divergence) were analyzed separately - subsegmental QCA analysis was performed according to European Bifurcation Club (EBC) Consensus [11]. The following parameters were calculated: lesion length, reference vessel diameter (RVD), minimal lumen diameter (MLD), \% diameter stenosis (\%DS), acute lumen gain and LLL before and after stent implantation and/or on follow-up. All reference diameters were measured $5 \mathrm{~mm}$ from the end of angiographically visible plaque in all 3 segments of bifurcation without use of interpolations (user defined reference diameters). Percent diameter stenosis (using parameters from each segment) was measured for each vessel segment separately using the following formula: \%DS $=[1-(\mathrm{MLD} /$ $/$ RVD) $] \times 100 \%$ [12]

\section{Statistical analysis}

Continuous variables were presented as mean \pm standard deviation. Categorical data were presented as numbers (\%). Continuous variables were compared using an unpaired Student t test, and categorical data using the $\chi^{2}$ test or Fisher exact test, as appropriate. If distribution was not normal, Wilcoxon signed-rank tests and Mann-Whitney U-tests were used. $\mathrm{P}$ values of $<0.05$ were considered statistically significant. Also, univariate and multivariate logistic regression analyses were performed. Statistical analysis was performed using R 3.0.2 for OS (R Foundation, Vienna, Austria).

\section{Results}

\section{Baseline clinical characteristics}

Between January 2012 and January 2014, a total of 152 patients were enrolled of whom 158 coronary bifurcation lesions were treated. The mean age was $62.6 \pm 9.11$ years and women had a share of $26.3 \%$ in the population. Most patients had stable CAD $(67.1 \%)$, arterial hypertension $(82.2 \%)$ and dyslipidemia (88.2\%). The detailed data are presented in Table 1.

\section{Angiographic and procedural characteristics}

The bifurcation lesions were most frequently located in left anterior descending artery; $50 \%$; Fig. 1A), and true bifurcations (Medina type 1,1,1;
Table 1. Demographics $(n=152)$.

\begin{tabular}{lc}
\hline & No. of patients (\%) \\
\hline Age & $62.6 \pm 9.11$ \\
Women & $40(26.3 \%)$ \\
Stable CAD & $102(67.1 \%)$ \\
NSTE-ACS & $50(32.9 \%)$ \\
Hypertension & $125(82.2 \%)$ \\
Dyslipidemia & $134(88.2 \%)$ \\
Diabetes type 2 & $43(28.3 \%)$ \\
Prior MI & $65(42.8 \%)$ \\
Prior PCl & $68(44.7 \%)$ \\
Coronary artery bypass graft & $16(10.5 \%)$ \\
Lower extremity artery disease & $12(7.9 \%)$ \\
Carotid artery disease & $6(3.9 \%)$ \\
Chronic kidney disease & $12(7.9 \%)$ \\
Smoking & $54(35.5 \%)$ \\
\hline
\end{tabular}

CAD - coronary artery disease; NSTE-ACS - non-ST-elevation acute coronary syndrome; $\mathrm{MI}$ - myocardial infarction; $\mathrm{PCl}-$ percutaneous coronary intervention

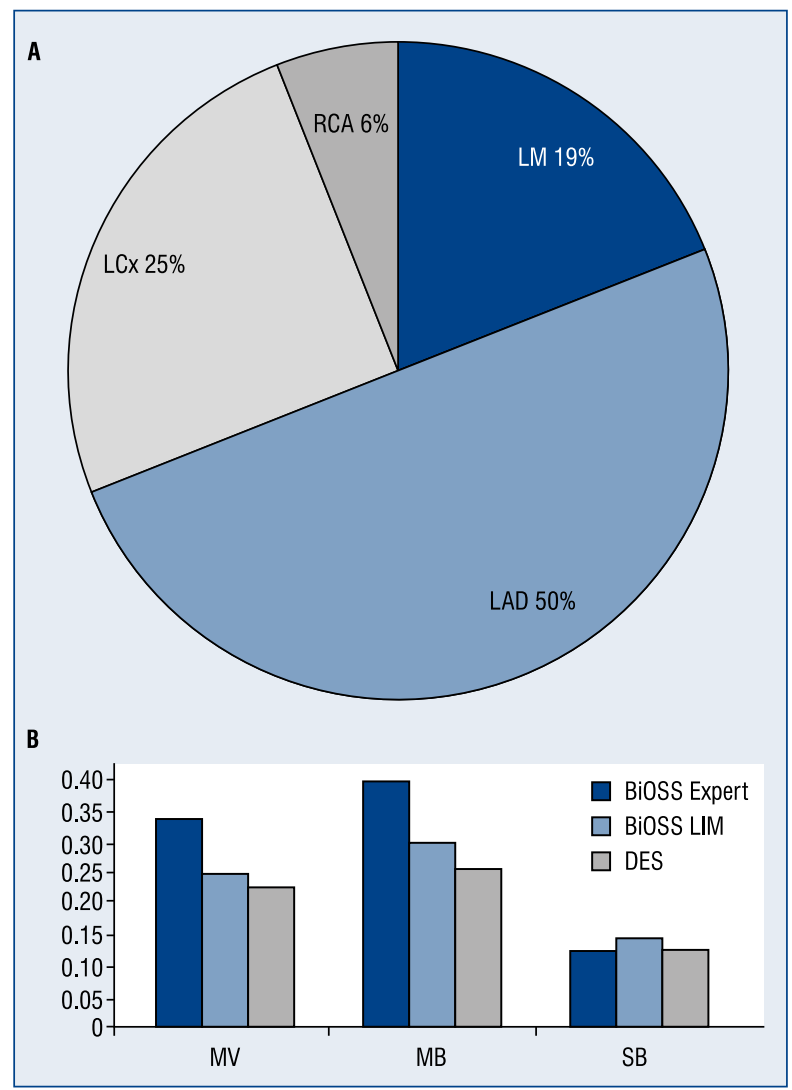

Figure 1. A. Lesion location; B. Late lumen loss in $\mathrm{mm}$; LAD — left anterior descending artery; LCx - left circumflex artery; LM - left main coronary artery; RCA right coronary artery; $\mathrm{MB}$ - main branch; $\mathrm{MV}$ - main vessel; SB - side branch. 
Table 2. Lesion and stent characteristics ( $n=158$ ).

\begin{tabular}{lc}
\hline Lesion characteristics & $\begin{array}{c}\text { No. of lesions } \\
(\%)\end{array}$ \\
\hline Medina type & $34(21.5 \%)$ \\
1.1 .1$. & $22(13.9 \%)$ \\
1.1 .0$. & $32(20.2 \%)$ \\
1.0 .1$. & $36(22.8 \%)$ \\
0.1 .1$. & $21(13.3 \%)$ \\
1.0 .0$. & $13(8.2 \%)$ \\
0.1 .0 & \\
Lesion type & $0(0 \%)$ \\
A & $76(48.1 \%)$ \\
B1 & $50(31.6 \%)$ \\
B2 & $32(20.3 \%)$ \\
C & \\
Stent type & $59(37.3 \%)$ \\
Regular DES: & $18(11.4 \%)$ \\
Paclitaxel eluting DES & $19(12.0 \%)$ \\
Sirolimus eluting DES & $22(13.9 \%)$ \\
Everolimus eluting DES & $99(62.7 \%)$ \\
Dedicated bifurcation stent: & $20(12.7 \%)$ \\
BiOSS Expert (paclitaxel eluting) & $79(50 \%)$ \\
BiOSS LIM (sirolimus eluting) &
\end{tabular}

DES - drug eluting stent

$1,0,1$ or $0,1,1)$ stand for $102(64.6 \%)$ treated lesions. Only DES were deployed among which most frequently dedicated bifurcation stents were used $(\mathrm{n}=99,62.7 \%)$, followed by $\mathrm{rDES}(\mathrm{n}=59$, $37.3 \%$ ) (Table 2).

The main procedural aspects are presented in Table 3. All stents were successfully implanted. In 10 cases the additional stent was implanted into the $\mathrm{SB}$, mainly using T-and-protrusion (TAP) or culotte techniques. In the dedicated bifurcation stent subgroup SB was stented in $4(4.04 \%)$ cases due to significant stenosis and large diameter, whereas in regular DES $6(10.2 \%)$ cases were registered due to significant stenosis in a large SB $(\mathrm{n}=4)$ or due to flow limiting dissection $(n=2)$. Final kissing balloon was applied in $24.1 \%$ of procedures.

\section{Clinical outcomes}

There were 5 (3.2\%) cases of periprocedural MI due to transient SB occlusion. Additionally, there were $9(5.7 \%)$ cases of in-hospital increase of TnI level (max $1.8 \mathrm{ng} / \mathrm{mL})$ - however, they were asymptomatic/without electrocardiogram changes which did not require repeated angiography (MI type 4 a criteria not met).
Table 3. Procedural characteristics $(n=158)$.

\begin{tabular}{lc}
\hline Parameter & $\begin{array}{c}\text { No of lesions } \\
(\%)\end{array}$ \\
\hline Successful implantation & $158(100 \%)$ \\
Main vessel predilatation & $130(82.3 \%)$ \\
Side branch predilatation & $115(72.8 \%)$ \\
Both branches predilatation & $6(3.8 \%)$ \\
Regular DES nominal & $3.15 \pm 0.60 \times$ \\
parameters [mm] & $20.69 \pm 8.81$ \\
Dedicated bifurcation stent & $3.63 \pm 0.36 \times$ \\
nominal parameters & $2.95 \pm 0.35 \times$ \\
(proximal diameter $x$ distal & $18.42 \pm 3.34$ \\
diameter $x$ length) [mm] & $38(24.1 \%)$ \\
Side branch postdilatation & $7(4.4 \%)$ \\
Proximal optimization technique & $38(24.1 \%)$ \\
Final kissing balloon & $10(6.3 \%)$ \\
Additional stent in side branch & $12.5 \pm 8$ \\
Fluoroscopy time [min] & $161 \pm 93$ \\
Contrast volume [mL] & $7 \% / 93 \%$ \\
Vascular access femoral/radial & $100 \% / 0 \%$ \\
Guiding catheter 6 F/7 F & $\mathrm{n}=10(\%)$ \\
Double-stent technique: & $1(10 \%)$ \\
T-stenting & $4(40 \%)$ \\
TAP & $1(10 \%)$ \\
Mini-crush & $4(40 \%)$ \\
Culotte & \\
\hline DES - drug-eluting stent; TAP - T-and-protrusion technique \\
\end{tabular}

Clinical follow-up data at 12 months were available in all patients (Table 4). The MACE incidence was $11.2 \%(\mathrm{n}=17)$ and it was similar between the dedicated bifurcation stent subgroup and the regular DES subgroup, $11.5 \%$ and $10.5 \%$, respectively. There were 5 cardiac deaths, in which 4 were sudden cardiac deaths and one death was caused by complications of MI. The TLR rate was 7.9\% $(\mathrm{n}=12)$. No definite in-stent thrombosis was registered, but one cannot exclude in-stent thrombosis since there were 4 sudden cardiac deaths. Further analyses are presented as Supplementary Table 1 and $\mathbf{2}$ (see journal website) - they describe clinical outcomes in diabetic and left main subgroups, respectively.

Quantitative coronary angiography analysis

Angiographic follow-up at 12 months was available in $78(51.3 \%)$ patients, of whom 34 had BiOSS Expert ${ }^{\circledR}$ stent implanted, 28 - BiOSS LIM ${ }^{\circledR}$ and 16 - regular DES. The late lumen loss values in separate segments of coronary bifurcation are presented in the Figure 1B. Similar LLL values were obtained in rDES and BiOSS LIM ${ }^{\circledast}$ groups, 
Table 4. Clinical results.

\begin{tabular}{|c|c|c|c|}
\hline & Whole population $(\mathrm{n}=152$ ) & DBS (n = 95) & Regular DES ( $n=57$ ) \\
\hline MACE & $17(11.2 \%)$ & $11(11.5 \%)$ & $6(10.5 \%)$ \\
\hline All-cause death & $9(5.9 \%)$ & $5(5.3 \%)$ & $4(7.0 \%)$ \\
\hline Cardiac death & $5(3.3 \%)$ & $2(2.1 \%)$ & $3(5.3 \%)$ \\
\hline Myocardial infarction & $5(3.3 \%)$ & $3(3.2 \%)$ & $2(3.5 \%)$ \\
\hline Stent thrombosis & $0(0 \%)$ & $0(0 \%)$ & $0(0 \%)$ \\
\hline Target lesion revascularization & $12(7.9 \%)$ & $7(7.4 \%)$ & $5(8.8 \%)$ \\
\hline
\end{tabular}

MACE - major adverse cardiovascular events; DBS — dedicated bifurcation stents; DES — drug eluting stent

Table 5. Logistic regression for major adverse cardiovascular events.

\begin{tabular}{|c|c|c|c|c|}
\hline \multirow[t]{2}{*}{ Variate } & \multicolumn{2}{|c|}{ Univariate analysis } & \multicolumn{2}{|c|}{ Multivariate analysis } \\
\hline & OR (95\% Cl) & $\mathbf{P}$ & OR $(95 \% \mathrm{Cl})$ & $\mathbf{P}$ \\
\hline BiOSS vs. DES & $0.918(0.547-1.247)$ & 0.547 & & \\
\hline BiOSS Expert vs. DES & $1.111(0.650-1.831)$ & 0.568 & & \\
\hline BiOSS LIM vs. DES & $0.714(0.665-1.120)$ & 0.341 & & \\
\hline Sex: female vs. male & $0.747(0.670-1.123)$ & 0.789 & & \\
\hline Age [increase per 1 year] & $0.814(0.684-1.336)$ & 0.238 & & \\
\hline NSTE-ACS & $2.180(1.560-2.446)$ & 0.008 & $1.801(1.391-3.150)$ & 0.044 \\
\hline Arterial hypertension & $1.361(0.775-2.562)$ & 0.345 & & \\
\hline Diabetes mellitus & $1.254(0.881-1.565)$ & 0.258 & & \\
\hline Dyslipidemia & $1.127(0.454-1.665)$ & 0.456 & & \\
\hline Prior MI & $1.742(0.910-2.774)$ & 0.412 & & \\
\hline Prior $\mathrm{PCl}$ & $1.113(0.712-1.975)$ & 0.651 & & \\
\hline Coronary artery bypass graft & $1.343(0.763-3.129)$ & 0.345 & & \\
\hline Chronic kidney disease & $1.232(0.447-2.002)$ & 0.782 & & \\
\hline Smoking & $1.468(0.751-1.802)$ & 0.753 & & \\
\hline True bifurcation & $2.279(1.114-4.751)$ & 0.021 & $1.758(1.114-2.452)$ & 0.035 \\
\hline LM bifurcation & $1.238(1.110-3.272)$ & 0.019 & $1.831(0.893-2.318)$ & 0.441 \\
\hline MV predilatation & $1.701(1.443-3.678)$ & 0.009 & & \\
\hline SB predilatation & $1.315(0.822-2.215)$ & 0.245 & & \\
\hline SB stenting & $1.360(0.985-2.642)$ & 0.348 & & \\
\hline Final kissing balloon & $0.501(0.396-0.950)$ & 0.038 & $0.401(0.246-0.820)$ & 0.021 \\
\hline Proximal optimization technique & $1.101(0.656-1.352)$ & 0.392 & & \\
\hline
\end{tabular}

OR - odds ratio; $\mathrm{CI}$ - confidence interval; DES — drug eluting stents; NSTE-ACS — non-ST-elevation acute coronary syndrome; MI - myocardial infarction; $\mathrm{PCl}$ - percutaneous coronary intervention; $\mathrm{LM}$ - left main; $\mathrm{MV}$ - main vessel; SB — side branch

whereas BiOSS Expert ${ }^{\circledR}$ group characterized a slightly larger neointima growth. Also, when comparing LLL value significant differences in proximal MV and in distal MV, but not in SB, were observed.

\section{Logistic regression analysis}

Results of logistic regression analyses are presented in Table 5 and Table 6 for MACE and TLR, respectively. Regarding MACE rate, NSTE-ACS and true bifurcation were associated with worse clinical outcome, whereas FKB was associated with better clinical outcome. Similar results were obtained when the TLR rate was analyzed.

\section{Discussion}

The main findings of this study are: 1 ) coronary bifurcations were mainly treated with 1 stent (PTS 
Table 6. Logistic regression for target lesion revascularization.

\begin{tabular}{|c|c|c|c|c|}
\hline \multirow[t]{2}{*}{ Variate } & \multicolumn{2}{|c|}{ Univariate analysis } & \multicolumn{2}{|c|}{ Multivariate analysis } \\
\hline & OR (95\% CI) & $\mathbf{P}$ & OR (95\% Cl) & $\mathbf{P}$ \\
\hline BiOSS vs. DES & $1.018(0.537-1.237)$ & 0.623 & & \\
\hline BiOSS Expert vs. DES & $1.211(0.609-2.001)$ & 0.755 & & \\
\hline BiOSS LIM vs. DES & $0.814(0.615-1.760)$ & 0.188 & & \\
\hline Sex: female vs. male & $0.647(0.450-0.993)$ & 0.046 & & \\
\hline Age [increase per 1 year] & $0.694(0.523-1.416)$ & 0.623 & & \\
\hline NSTEMI/UA & $2.080(1.640-3.566)$ & 0.007 & $1.921(1.231-3.120)$ & 0.004 \\
\hline Arterial hypertension & $1.411(0.675-3.102)$ & 0.212 & & \\
\hline Diabetes mellitus & $1.244(0.651-1.675)$ & 0.498 & & \\
\hline Dyslipidemia & $1.317(0.732-1.455)$ & 0.578 & & \\
\hline Prior MI & $1.412(0.830-2.414)$ & 0.243 & & \\
\hline Prior $\mathrm{PCl}$ & $1.223(0.872-2.275)$ & 0.878 & & \\
\hline Coronary artery bypass graft & $1.523(0.863-2,729)$ & 0.465 & & \\
\hline Chronic kidney disease & $1.412(0.767-2.122)$ & 0.619 & & \\
\hline Smoking & $1.218(0.751-1.802)$ & 0.773 & & \\
\hline True bifurcation & $2.339(1.324-3.671)$ & 0.019 & $1.988(1.442-4.172)$ & 0.031 \\
\hline LM bifurcation & $1.328(1.115-3.672)$ & 0.042 & $1.421(0.893-2.218)$ & 0.655 \\
\hline MV predilatation & $1.211(1.013-3.128)$ & 0.032 & & \\
\hline SB predilatation & $1.215(0.782-1.785)$ & 0.166 & & \\
\hline SB stenting & $1.210(0.765-3.542)$ & 0.174 & & \\
\hline Final kissing balloon & $0.721(0.496-0.980)$ & 0.037 & $0.798(0.226-0.960)$ & 0.041 \\
\hline Proximal optimization technique & $0.941(0.508-1.122)$ & 0.534 & & \\
\hline
\end{tabular}

OR — odds ratio; $\mathrm{Cl}$ - confidence interval; DES — drug eluting stents; NSTEMI/UA — non-ST-elevation myocardial infarction/unstable angina; $\mathrm{MI}$ — myocardial infarction; $\mathrm{PCl}$ — percutaneous coronary intervention; LM — left main; MV — main vessel; SB — side branch

strategy), 2) 1-year MACE and TLR rates were $11.2 \%$ and $7.9 \%$, respectively, 3 ) clinical outcomes for dedicated bifurcation stents BiOSS and regular DES were similar, 4) optimization techniques (FKB, POT) were rarely used.

In this study the population was severely diseased, with rates of diabetes $(28.3 \%)$, prior MI $(42.8 \%)$ and prior PCI (44.7\%) which is higher than in other studies assessing bifurcation treatment, $11-25.7 \%, 19.5-46 \%$ and $11.3-37.1 \%$ [13-17] respectively.

In recent years, a series of studies helped to characterize the coronary bifurcation anatomy, and geometric relations linking MV and SB were expressed as mathematical models such as Murray's, Finet's or Huo-Kassab's laws [13, 14, 18]. To some extent as a result, EBC recommends PTS as the standard strategy for treatment of coronary bifurcation. Although there are lesions for which PTS is not the optimal approach, the need for an alternative strategy is relatively rare in most cases [11]. Results obtained in this registry were concordant with these recommendations. Almost all bifurcations $(93.7 \%)$ were treated with PTS strategy, and what is important is that $64.5 \%$ of cases were true bifurcations. As proven earlier, PTS strategy ensured the best angiographic and clinical outcomes in the majority of studies [11]. Moreover, Kim et al. [15] as well as others showed that a 1 -stent technique was better than a 2 -stent technique $[15,16]$.

Only 10 cases required a two-stent technique, mainly performed with TAP and culotte. Worth stressing is the fact that all culotte procedures were performed in distal left main with the deployment of two BiOSS LIM ${ }^{\circledR}$ stents as described previously [17].

Predilatation of MV prior to stenting is the common approach, whereas routine SB dilation is unnecessary. Nevertheless, in the presence of severe SB ostial stenosis it should be considered. We performed MV predilatations in $82.3 \%$ cases and SB predilatations in $72.8 \%$. We had in mind that potential advantages of SB dilatation include 
increased ostial SB lumen, facilitated rewiring of the SB after stenting and avoiding rewiring and post-dilatation of the SB after implantation of the MV stent [19].

Appropriate stent apposition in the proximal $\mathrm{MV}$ is achieved by POT, which is performed by dilating the proximal MV stent from the proximal stent edge to just proximal to the carina, using a short oversized balloon. POT facilitates SB access, reduces risk of accidental abluminal rewiring, lowers the risk of stent distortion by catheter collision, and enhances scaffolding at the SB ostium. Thus, POT should be considered a standard step in the bifurcation treatment. Also, FKB is the technique which optimizes the procedure [11]. Unfortunately, in our paper rates of POT and FKB were low, $4.4 \%$ and $24.1 \%$, respectively. This could have been caused by the fact that only recently POT is strongly recommended by $\mathrm{EBC}$, and in case of $\mathrm{FKB}$ - that only rarely a 2 -stent technique was used where $\mathrm{FKB}$ is obligatory. Also, in $62.7 \%$ of cases dedicated bifurcation BiOSS stents were implanted. Theoretically, the stepped design of the $\mathrm{BiOSS}^{\oplus}$ delivery balloon was to ensure FKB- and POT-like effects, thus allowing operators to frequently omit this part of the procedure. However, operators firmly believed that the $\mathrm{BiOSS}^{\circledR}$ construction ensures those effects. As was shown in POLBOS I trial the lack of FKB/POT was associated with the worse clinical outcome and the trend in larger late lumen loss values, whereas in the NORDIC 3 study it was proved that FKB reduced angiographic side branch restenosis, especially in patients with true bifurcation lesions $[20,21]$. These findings were confirmed in the MITO Registry [22]. Also worth stressing, is the fact that the negative impact of true bifurcation and positive impact of FKB on MACE and TLR rates were also confirmed in the present logistic regression analyses.

Nevertheless, the results obtained in the mentioned registry (MACE $11.2 \%$, TLR 7.9\%) were comparable or even better than in other clinical trials assessing coronary bifurcation treatment such as POLBOS I [21], POLBOS II [23] or EBC TWO [24].

As a kind of innovation in this study dedicated bifurcation stents were used. As was already mentioned dedicated bifurcation stents were used in $62.7 \%$ of cases. In recent years bifurcation dedicated stents were developed but majority of them did not enter routine clinical practice. In the Tryton trial, 704 patients with non-left main, true coronary bifurcation lesions were randomized to a Tryton-facilitated culotte technique or to a PTS with an everolimus-eluting stent [25]. At 9 months, the primary endpoint (target vessel failure) was $17.4 \%$ in the Tryton group compared with $12.8 \%$ in the PTS group. Obtained results showed that safety and efficacy reached by PTS with the latest-generation DES make the role of dedicated stents for non-left main lesions quite limited. Based on this, the EBC consensus was in favor of considering distal left main treatment due to its specific anatomic complexity, which at least in theory may benefit from technical improvements of dedicated stents [11]. This position is supported by published reports [9, 26-28].

\section{Limitations of the study}

This registry has several limitations that should be acknowledged. First of all the sample size was relatively small and no sample size calculation was performed. Other limitations of this study are its non-randomized manner as well as other drawbacks of registry studies. Also, the variety of rDES use, the lack of intravascular ultrasound, use and the relatively low diabetes type 2 rate could be treated as drawbacks.

\section{Conclusions}

Percutaneous coronary bifurcation treatment is safe and effective procedure, and provisional T-stenting is the preferred technique. Both regular DES as well as dedicated bifurcation stents BiOSS Expert $^{\circledast}$ and BiOSS LIM ${ }^{\circledR}$ enabled a simple and fast bifurcation treatment option with a single stent and with comparable MACE and TLR rates.

\section{Conflict of interest: None declared}

\section{References}

1. Chatzizisis YS, Jonas M, Coskun AU, et al. Prediction of the localization of high-risk coronary atherosclerotic plaques on the basis of low endothelial shear stress: an intravascular ultrasound and histopathology natural history study. Circulation. 2008; 117(8): 993-1002, doi: 10.1161/CIRCULATIONAHA.107.695254, indexed in Pubmed: 18250270.

2. Song YB, Hahn JY, Yang JH, et al. Differential prognostic impact of treatment strategy among patients with left main versus non-left main bifurcation lesions undergoing percutaneous coronary intervention: results from the COBIS (Coronary Bifurcation Stenting) Registry II. JACC Cardiovasc Interv. 2014; 7(3): 255-263, doi: 10.1016/j.jcin.2013.11.009, indexed in Pubmed: 24529936.

3. Peña-Duque MA, Romero-Ibarra JL, Gaxiola-Macías MB, et al. Coronary atherosclerosis and interventional cardiology. Arch Med Res. 2015; 46(5): 372-378, doi: 10.1016/j.arcmed.2015.06.005, indexed in Pubmed: 26117516.

4. Zamani P, Kinlay S. Long-term risk of clinical events from stenting side branches of coronary bifurcation lesions with drug- 
eluting and bare-metal stents: an observational meta-analysis. Catheter Cardiovasc Interv. 2011; 77(2): 202-212, doi: 10.1002/ /ccd.22750, indexed in Pubmed: 20824754.

5. Chen L, Fan L, Luo Y, et al. Ex vivo mono-ring technique simplifies culotte stenting for treatment of true bifurcation lesions: Insights from bench testing and clinical application. Cardiol J. 2016; 23(6): 673-684, doi: 10.5603/CJ.a2016.0054, indexed in Pubmed: 27515483.

6. Jenei C, Balogh E, Szabó GT, et al. Wall shear stress in the development of in-stent restenosis revisited. A critical review of clinical data on shear stress after intracoronary stent implantation. Cardiol J. 2016; 23(4): 365-373, doi: 10.5603/CJ.a2016.0047, indexed in Pubmed: 27439365.

7. Ng AKY, Jim MH. Percutaneous coronary intervention for bifurcation: how can we outperform the provisional strategy? Clin Cardiol. 2016; 39(11): 684-691, doi: 10.1002/clc.22581, indexed in Pubmed: 27556582.

8. Thygesen K, Alpert JS, Jaffe AS, et al. Third universal definition of myocardial infarction. Circulation. 2012; 126(16): 2020-2035, doi: 10.1161/cir.0b013e31826e1058.

9. Bil J, Gil RJ, Vassilev D, et al. Dedicated bifurcation paclitaxel-eluting stent BiOSS Expert ${ }^{\circledR}$ in the treatment of distal left main stem stenosis. J Interv Cardiol. 2014; 27(3): 242-251, doi: 10.1111/joic.12119, indexed in Pubmed: 24708143.

10. Gil RJ, Bil J, Vassilev D. The BiOSS stent. EuroIntervention. 2015; 11(Suppl V): V153-V154.

11. Lassen JF, Holm NR, Banning A, et al. Percutaneous coronary intervention for coronary bifurcation disease: 11th consensus document from the European Bifurcation Club. EuroIntervention. 2016; 12(1): 38-46, doi: 10.4244/EIJV12I1A7, indexed in Pubmed: 27173860.

12. Bil J, Gil RJ, Kern A, et al. Novel sirolimus-eluting stent Prolim ${ }^{\circledR}$ with a biodegradable polymer in the all-comers population: one year clinical results with quantitative coronary angiography and optical coherence tomography analysis. BMC Cardiovasc Disord. 2015; 15: 150, doi: 10.1186/s12872-015-0139-5, indexed in Pubmed: 26573577.

13. Finet G, Gilard M, Perrenot B, et al. Fractal geometry of arterial coronary bifurcations: a quantitative coronary angiography and intravascular ultrasound analysis. EuroIntervention. 2008; 3(4): 490-498, indexed in Pubmed: 19736093.

14. Huo Y, Finet G, Lefèvre T, et al. Optimal diameter of diseased bifurcation segment: a practical rule for percutaneous coronary intervention. EuroIntervention. 2012; 7(11): 1310-1316, doi: 10.4244/EIJV7I11A206, indexed in Pubmed: 22433194.

15. Kim WJ, Kim YH, Park DW, et al. Comparison of single- versus two-stent techniques in treatment of unprotected left main coronary bifurcation disease. Catheter Cardiovasc Interv. 2011; 77(6): 775-782, doi: 10.1002/ccd.22915, indexed in Pubmed: 21520380.

16. Chen SL, Zhang Y, Xu Bo, et al. Five-year clinical follow-up of unprotected left main bifurcation lesion stenting: one-stent versus two-stent techniques versus double-kissing crush technique. EuroIntervention. 2012; 8(7): 803-814, doi: 10.4244/EIJV8I7A123, indexed in Pubmed: 23171801.

17. Vassilev D, Mateev H, Alexandrov A, et al. Double bifurcation optimization stent system technique for left main stenosis. J Interv Cardiol. 2014; 27(6): 570-573, doi: 10.1111/joic.12155, indexed in Pubmed: 25224296.
18. Murray CD. The physiological principle of minimum work applied to the angle of branching of arteries. J Gen Physiol. 1926; 9(6): 835-841, indexed in Pubmed: 19872299.

19. Pan M, Medina A, Romero M, et al. Assessment of side branch predilation before a provisional T-stent strategy for bifurcation lesions. A randomized trial. Am Heart J. 2014; 168(3): 374-380, doi: 10.1016/j.ahj.2014.05.014, indexed in Pubmed: 25173550.

20. Niemelä M, Kervinen K, Erglis A, et al. Randomized comparison of final kissing balloon dilatation versus no final kissing balloon dilatation in patients with coronary bifurcation lesions treated with main vessel stenting: the Nordic-Baltic Bifurcation Study III. Circulation. 2011; 123(1): 79-86, doi: 10.1161/CIRCULATIONAHA.110.966879, indexed in Pubmed: 21173348.

21. Gil RJ, Bil J, Džavík V, et al. Regular drug-eluting stent vs dedicated coronary bifurcation bioss expert stent: multicenter open-label randomized controlled POLBOS I trial. Can J Cardiol. 2015; 31(5): 671-678, doi: 10.1016/j.cjca.2014.12.024, indexed in Pubmed: 25828372.

22. Takagi K, Ielasi A, Basavarajaiah S, et al. The impact of main branch restenosis on long term mortality following drug-eluting stent implantation in patients with de novo unprotected distal left main bifurcation coronary lesions: the Milan and NewTokyo (MITO) Registry. Catheter Cardiovasc Interv. 2014; 84(3): 341-348, doi: 10.1002/ccd.25178, indexed in Pubmed: 24038911.

23. Gil RJ, Bil J, Grundeken MJ, et al. Regular drug-eluting stents versus the dedicated coronary bifurcation sirolimus-eluting BiOSS LIM $^{\varpi}$ stent: the randomised, multicentre, open-label, controlled POLBOS II trial. EuroIntervention. 2016; 12(11): e1404-e1412, doi: 10.4244/EIJY15M11_11, indexed in Pubmed: 26600564 .

24. Hildick-Smith D, Behan MW, Lassen JF, et al. The EBC TWO Study (European Bifurcation Coronary TWO): A Randomized Comparison of Provisional T-Stenting Versus a Systematic 2 Stent Culotte Strategy in Large Caliber True Bifurcations. Circ Cardiovasc Interv. 2016; 9(9), doi: 10.1161/CIRCINTERVENTIONS.115.003643, indexed in Pubmed: 27578839.

25. Généreux P, Kumsars I, Lesiak M, et al. A randomized trial of a dedicated bifurcation stent versus provisional stenting in the treatment of coronary bifurcation lesions. J Am Coll Cardiol. 2015; 65(6): 533-543, doi: 10.1016/j.jacc.2014.11.031, indexed in Pubmed: 25677311.

26. Magro M, Girasis C, Bartorelli AL, et al. Acute procedural and six-month clinical outcome in patients treated with a dedicated bifurcation stent for left main stem disease: the TRYTON LM multicentre registry. EuroIntervention. 2013; 8(11): 1259-1269, doi: 10.4244/EIJV8I11A194, indexed in Pubmed: 23538155.

27. Briguori C, Visconti G, Donahue M, et al. The STENTYS ${ }^{\circledR}$ paclitaxel-eluting stent in the treatment of unprotected distal left main. Catheter Cardiovasc Interv. 2015; 86(3): E131-E139, doi: 10.1002/ccd.25874, indexed in Pubmed: 25639945.

28. Gil RJ, Bil J, Grundeken MJ, et al. Long-term effectiveness and safety of the sirolimus-eluting BiOSS LIM ${ }^{\circledast}$ dedicated bifurcation stent in the treatment of distal left main stenosis: an international registry. EuroIntervention. 2016; 12(10): 1246-1254, doi: 10.4244/EIJY15M10_05, indexed in Pubmed: 26465375 . 